\title{
Normalized uptake fraction: an alternative for metabolic quantification
}

\begin{abstract}
Introduction: Functional imaging with ${ }^{18} \mathrm{~F}$-FDG PET/CT has become a valuable tool in Oncology. Standardized uptake value represents a semi-quantitative approach in PET in order to have an easy way to evaluate tissues metabolic rate. Despite the recommendations for its inclusion in clinical reports, its calculation is affected by multiple variables making difficult to compare exams from different labs or to use it in radiotherapy planning. Our goal was the development of an alternative methodology of quantification that despite remaining semi-quantitative is less operator's dependent.
\end{abstract}

Material and methods: We studied 32 women with cervical cancer in stage IIB, IIIA and IIIB that were scanned with ${ }^{18} \mathrm{~F}$-FDG PET/CT previously to treatment. Additionally, one woman had an evaluation post treatment, and another one was also evaluated 25 months after treatment terminus. Images were included in Velocity $\mathrm{AI} \rightarrow$ and the results of an independent and blind evaluation by two experts including the contouring of gross tumor volume were compared. Images were also included in MEVISLAB ${ }^{\circledR}$ platform, an image processing and visualization software, and we obtained parametric images of the distribution of, what we called, normalized uptake fraction (FCN). Visualization of the FCN images was lower bounded by taking into account the activity of quadriceps muscle. Representative clinical cases were selected to describe our image results.

Results and conclusion: We obtained an independent visualization method, allowing a new approach in quantification of metabolic imaging. There is evidence that, through visual navigation in CT-mode but mainly in PET/CT-mode after application of FCN, we were allowed to analyze quantification images of PET/CT, from different patients and different equipment's, without the controversial ROI or VOI definition and measurement of respective SUV. For radiotherapy planning the proposed method seems to improve the determination of biological target volume (BTV).

Keywords: metabolic quantification, ${ }^{18} \mathrm{~F}-\mathrm{FDG}$ PET/CT, normalized uptake fraction, volume of interest, gross tumor volume
Volume 4 Issue I - 2016

\author{
Paula P Alves, ${ }^{1,2}$ Augusto F Silva, ${ }^{3}$ Maria F \\ Botelho' \\ 'Radiotherapy Department, IPOCFG, Portugal \\ ${ }^{2}$ Biophysics Institute, Portugal \\ ${ }^{3}$ Electronics, Telecommunications and Informatics Department, \\ University of Aveiro, Portugal
}

Correspondence: Paula P Alves, Radiotherapy Department, IPOCFG, Avenida Bissaya Barreto, 98, 300I-65I Coimbra, Portugal,Tel 35I 919850040,Email ppinto.alves@gmail.com

Received: December 12, 2015 | Published: January 18, 2016
Abbreviations: ${ }^{18} \mathrm{~F}-\mathrm{FDG}$, 2-deoxy-2-( $\left.{ }^{18} \mathrm{~F}\right)$ fluoro-d-glucose; $\mathrm{PET} / \mathrm{CT}$, positron emission tomography/computed tomography; SUV, standard uptake value; FCN, normalized uptake fraction; $\mathrm{CT}$, computed tomography; ROI, region of interest VOI, volume of interest; BTV, biological target volume; H\&N, head and neck; TPS, treatment planning systems; EANM, european association of nuclear medicine; IMRT, intensity modulated radiotherapy; VMAT, volumetric arc therapy; FIGO, international federation of gynecology and obstetrics; 3D-CRT, three-dimensional conformal radiotherapy; GTV, gross tumor volume

\section{Introduction}

PET/CT with ${ }^{18} \mathrm{~F}-\mathrm{FDG}$ has become a valuable tool in Oncology mainly in diagnosis, staging as well as in the therapeutic monitoring and radiotherapy planning for lung cancer, $\mathrm{H} \& \mathrm{~N}$ cancer and lymphoma, which is translated by numerous publications. ${ }^{1-3}$ For the last decades radiotherapy has pursued great technological achievements with impact in treatment equipment, mainly in the linacs, but also in treatment planning systems (TPS). Those systems have new tools allowing radiation oncologists to obtain new images resulting from image fusion coming from different origin, using rigid or elastic algorithms of deformation and automatic or semi-automatic segmentation. For these reasons, the integration of functional image in radiotherapy planning practice is attractive, and more and more used in a daily base. Visser et al. ${ }^{4}$ in their studies have mentioned that quantification of metabolic activity is a big challenge in PET as it means that a "raw signal of PET can be transferred into absolute activity concentrations" of the radiopharmaceutical, with its units of measure.4 Quantification of metabolic image, in the present stateof- the-art, is performed by the standardized uptake values, known as SUV. The referred quantification is, in fact, a semi-quantification, once it dependents of multiple bias, such as the detector calibration factor, the time of acquisition, the image reconstruction methods including system resolution and the type of filters used, the size of the volume of interest (VOI), the glycemic patient status, among others. ${ }^{5}$ Recent EANM guidelines for tumor imaging emphasize de need of repeatability and reproducibility of data for quantification measures. ${ }^{6}$ Version 2.0 of the guidelines recommends the use of SUVmax sustaining that this value is the most independent from the observer, as it represents the highest uptake of radiotracer in the voxel. Discordant voices claim that hopefully this particular voxel must be included in the volume of interest (VOI), which is found by the observer itself, and there is again the observer dependence of this value. Others question is how can a voxel be representative of the metabolism of a tumoral volume. ${ }^{7}$ Additionally, Frey et al. ${ }^{8}$ mentioned that SUVmax is strongly dependent of the image noise and consequently dependent of reconstruction methodology. ${ }^{8,9}$ It seemed important to know if all the information provided by PET/CT with ${ }^{18} \mathrm{~F}-\mathrm{FDG}$ is achievable and helpful for radiation oncologist, who frequently receives exams from different labs and of different patients, 
all enrolled in the same sequence of events, for radiotherapy planning purposes. Cervical cancer is among the top four of cancer diagnosis in women worldwide. ${ }^{10}$

It is a foreseeable entity in respect of patterns of local invasiveness and dissemination and these characteristics respond for its behavior as a model of neoplastic malignant disease. The treatment of cervical cancer has changed over the time with an important landmark in the last decades, with the inclusion of concurrent chemotherapy along with external radiotherapy, ${ }^{11}$ mainly a cisplatin-based chemotherapy, using radiosensitizer concentrations. Nevertheless, toxicity remains a major and unsolved problem. Intensity modulated radiotherapy (IMRT) an advanced radiotherapy technique, is not the gold standard for treating the primary cervical lesion, because of the difficulty to establish the boundaries of the tumor, as it infiltrates the parametrium and soft tissues of side pelvic wall. ${ }^{12}$ Treatment of lymph nodes, mainly paraaortic lymph nodes, which occur in almost $30 \%$ of patients in stage III (FIGO) of the disease, with IMRT (with or without integrated boost), seems feasible and effective, with acceptable toxicity in small bowel and bone marrow ${ }^{13}$ when compared with $3 \mathrm{D}$-conformal radiotherapy (3D-CRT). Accurate target definition, in cervical cancer, is of major importance to unsure that there is no under-treatment and to limit the dose to surrounding normal tissues. ${ }^{14}$

\section{Materials and methods}

In order to answer the question mentioned above, if all the information provided by $\mathrm{PET} / \mathrm{CT}$ with ${ }^{18} \mathrm{~F}$-FDG (PET/CT) is achievable and helpful for radiation oncologist, we choose to study a group of 32 patients, after obtaining their permission, as Helsinki Convention determines. All the patients had histological evidence of invasive cervical carcinoma, 26 patients with squamous cell carcinoma and 6 patients with adenocarcinoma. Patients were classified in stage IIB (13 patients) and IIIB (19 patients), applying international classification of FIGO with indication for intensive radio chemotherapy. $15,16{ }^{18} \mathrm{~F}$-FDG PET/CT's were acquired pretreatment, and radiotherapy planning included functional images. ${ }^{18} \mathrm{~F}-\mathrm{FDG}$ $\mathrm{PET} / \mathrm{CT}$ were from two different labs. We obtained $32 \mathrm{PET} / \mathrm{CT}$ pretreatment, $1 \mathrm{PET} / \mathrm{CT}$ after external radiotherapy, one PET/CT during follow-up ( 25 months post treatment terminus) and 32 planning CT. All images of planning CT and PET/CT were included in Velocity Advanced Imaging (Velocity AI), a software that provides automated multi-modality image registration, among other planning tools, and 2 independent experts, in a blind study, made the contouring of gross tumor volume (GTV) from planning CT information, named as GTV$\mathrm{CT}$, and incorporating functional information, named as GTV-SUV, by the method of direct visualization, obtaining two sets of images each. The two sets of GTV's, from the observers, called observer A and observer B, were compared, analyzing intra -observer results and inter-observer results. Statistical analysis used Spearman coefficient and it was assumed an error type I of 0.05 for all the comparisons. It was our option to determine a correlation coefficient, since we could not measure agreement with kappa, as we did not categorize the variables GTV-CT and GTV-SUV. All PET/CT images were included in MEVISLAB software, software for visualization and processing of medical imaging, through an iconic visual programming paradigm, after the application of what we called a normalized uptake fraction per voxel (FCN, from Portuguese, Fracção de Captação Normalizada). The new methodology of quantification achieved (FCN) was applied to all images, and experts analyzed parametric images obtained. We selected the most representative clinical cases of locally advanced cervical cancer.

\section{Results and discussion}

The analysis of $34 \mathrm{PET} / \mathrm{CT}$ exams revealed great variability in acquisition methodology, SUV calculations, regions of interest (ROI) and clinical reports. Regarding inter-observer analysis, we observed a significant correlation between the GTV volumes determined by each observer (A and B), when delineated based on CT information (GTV$\mathrm{CT}, \mathrm{r}=0.936, \mathrm{p}<0.001)$ and when delineated considering PET/CT SUV information (GTV-SUV, $r=0.935, \mathrm{p}<0.001$ ). For each observer (intra-observer analysis), we found a correlation between both types of volumes that were delineated (GTV-CT and GTV-SUV), with a correlation value of 0.642 for observer $A(p<0.001)$ and of 0.593 for observer B $(\mathrm{p}<0.001)$, as seen in Table 1 .

Table I Gross Tumor Volume comparison; Volumes obtained by contouring in Velocity Al $\rightarrow$

\begin{tabular}{lll}
\hline Intra-observer analysis & & \\
Observer A & $\rho=0.642$ & $p<0.001$ \\
Observer B & $\rho=0.593$ & $p<0.001$ \\
Inter-observer analysis & & \\
GTV-CT & $\rho=0.936$ & $p<0.001$ \\
GTV-SUV & $\rho=0.935$ & $p<0.001$ \\
\hline
\end{tabular}

Abbreviations: GTV-CT: Gross Tumor Volume Obtained From CT Data; GTV-SUV: Gross Tumor Volume Obtained From Integration Of Functional Imaging.

When looking for differences between volumes obtained from CT data and volumes based on inputs from the integration of functional information into the final GTV, we did not manage to obtain differences with statistical significance. It seemed that functional information obtained from PET/CT had no impact in final delineation of gross tumor volume, for both experts, with their performances mostly sustained by morphological information from CT planning. At this point, we made a glance through metabolic quantification mentioned in clinical reports of our PET/CT exams. In fact, quantification was reported using maximum standardized uptake value (SUVmax), but SUVmax was not systematically recorded, and the values mentioned on clinical reports varied from a minimum of 3.7 to a maximum of 20.5 , with regions of interest frequently defined far from the primary lesion area, sometimes in epigastric region and others in thyroid area. Reporters seemed to make no completely confidence in SUV calculation, as it was their option not to reveal it to users, in a systematic way. In fact in 10 exams there was no reference of SUV at all. In this particular, our PET/CT images had great variability in acquisition methodology and it seemed necessary to develop a new and reliable procedure of metabolic quantification that could be applied to all images and simultaneously that could be simple and reproducible.

We started to calculate the FCN according to the expression: $\mathrm{FCN}=\mathrm{C} \square \mathrm{W}(\mathrm{g}) / \mathrm{Nt}$,

Where $\mathrm{C}$ represents counts/voxel, W the corporal mass in grams and $\mathrm{Nt}$ the total counts, and built a parametric image of its distribution. This type of normalization takes into account both the specificity of PET study and patient characteristics. Visualization of the FCN images was further conditioned by a lower bound obtained by the average FCN values at the thigh muscles. In practice, only the values above the thigh FCN were shown, translating the FDG high metabolic rate areas, coincident with cervical primary tumors and/ or related regional or peri-tumoral lymph nodes. In sequence, we included all PET/CT images in MEVISLAB software platform that allows visualization and processing of medical imaging, following an iconic visual programming. We managed to obtain a parametric 
image of FCN distribution per voxel, with visual optimization from a color table, tonal gradation, transparences/opacities and selection of views, including re- sampling with $3 \mathrm{D}$ reconstruction, allowing observer's interaction with images. Figure 1 represents PET/CT standard views (axial, coronal and sagittal) before and after FCN application. Although standard views, axial, coronal and sagittal, were obtained, it was de re-sampling with $3 \mathrm{D}$ reconstruction that was the major point of interest among the experts that reviewed these FCN parametric images. During the process, we found the necessity to resolve an interpretation constraint, due to the physiological retention of radiotracer in bladder, which is positioned anteriorly to the primary tumor area. For this purpose, bladder was delineated in CT with interactive segmentation with "livewire", in approximately 10 segments and a personalized bladder mask was constructed from 3D interpolation of sectional contours (Figure 2).
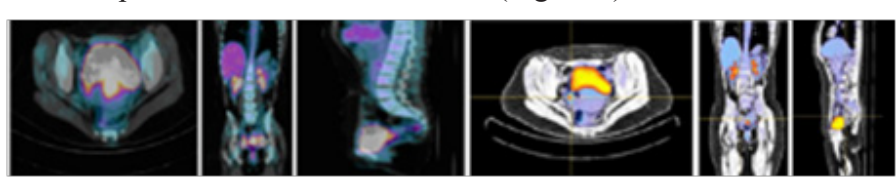

Figure I Axial, coronal and sagittal views before FCN application (left) and axial, coronal and sagittal views after FCN application (right).
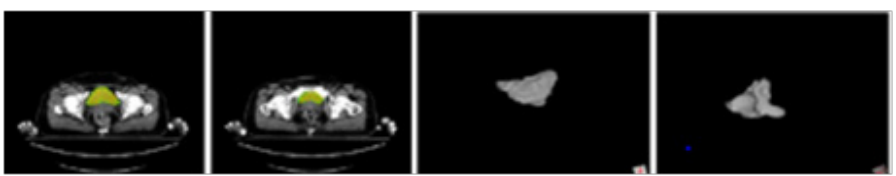

Figure 2 Bladder mask delineated on CT images constructed from 3D interpolation of sectional contours and posterior subtraction.

The new visualization method allowed the observers to select the best scenarios, in all cases, by interacting with the color scale and transparence/opacity tools, and by re-sampling with 3D reconstruction, with this option allowing a volumetric perspective of metabolic activity translated by FDG uptake, corresponding to gross tumor volume, with well defined boundaries, an issue of major interest for radiation oncologists and revealing all the attraction for the concept of biologic conformality, as postulated by Ling et al. ${ }^{17}$ (Figure 3 ). It was our decision to select representative clinical cases from our $34 \mathrm{PET} /$ $\mathrm{CT}$ and to obtain clinical interpretation of FCN parametric images, as follows:

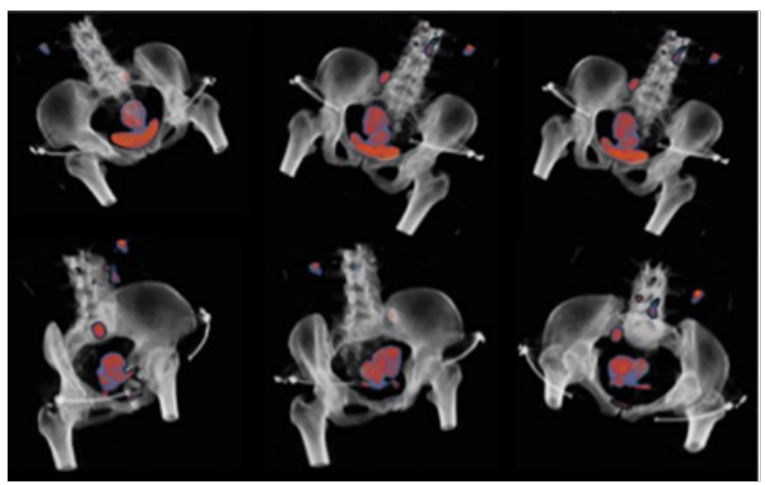

Figure $33 \mathrm{D}$ reconstruction after applying $\mathrm{FCN}$; on the top, 3 views without bladder occultation; on the bottom, same views with bladder mask interposition. Notice the cervical lesion and pelvic lymph node, as focus of high FDG uptake.

\section{Clinical case with pelvic lymph nodes and para-aortic lymph nodes}

Interpretation of images after application of $\mathrm{FCN}$ allowed the identification of an area of high metabolic uptake of ${ }^{18} \mathrm{~F}-\mathrm{FDG}$ between rectum and bladder, in relation with known cervical primary tumor. In sagittal view it is notorious a huge uptake focus adjacent to the fundus of uteri that might correspond to metastatic lymph node. It is evident, in the same view, a focus of hipermetabolic activity, with similar characteristics of the primary lesion, compatible with paraaortic lymph node (Figure 4). In 3D visualization mode, abdominalpelvic views allow to identify, with topographic precision uptake focus of ${ }^{18} \mathrm{~F}$ - FDG described above (Figure 5).
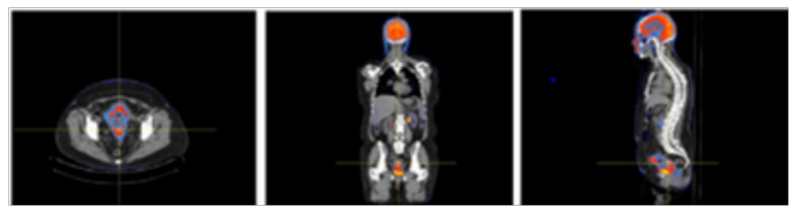

Figure 4 Axial, coronal and sagittal views after FCN application. Notice hypermetabolic focus adjacent to the uterus in sagittal view.
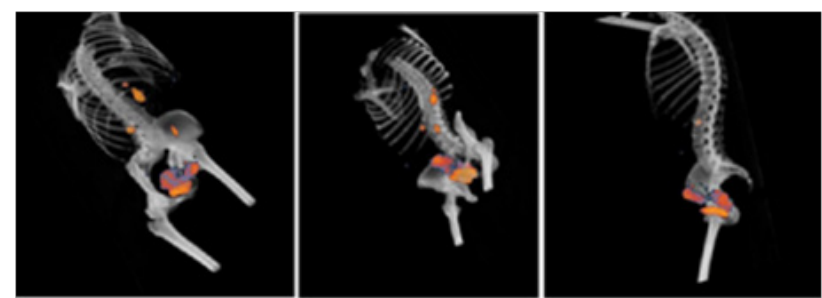

Figure 5 Views of 3D visualization mode. Notice hypermetabolic focus compatible with primary lesion, lesion in supra-uterine position and in lymph node in para-aortic area.

\section{Clinical case of unsuspected pelvic lymph node - the "proof of concept" "}

Interpretation of images, after application of $\mathrm{FCN}$, revealed a moderate ${ }^{18} \mathrm{~F}$-FDG uptake area between bladder and rectum, related to the primary cervical lesion. The bladder showed high uptake, because of physiological radiotracer retention, and bladder seemed to embrace tumoral area. Images in 3D visualization mode, with bladder occultation, showed 2 focus of high uptake of ${ }^{18} \mathrm{~F}$ - FDG, related with peri-tumoral lymph nodes and 2 others of uncertain significance. Notice that the 2 peri-tumoral foci were not visible/described in PET/ CT before FCN application (Figure 6). Comment: Clinical stage is not modified because of lymphatic dissemination but the evidence of metastatic lymph nodes implies new strategy of irradiation, by means of larger volumes of treatment, pondering advanced techniques for better dose distributions and dose escalation.15 Dose escalation in cervical cancer is a meaningful concept as this particular type of malignant disease is radio-resistant and its response to treatment is dose-dependent. ${ }^{16}$
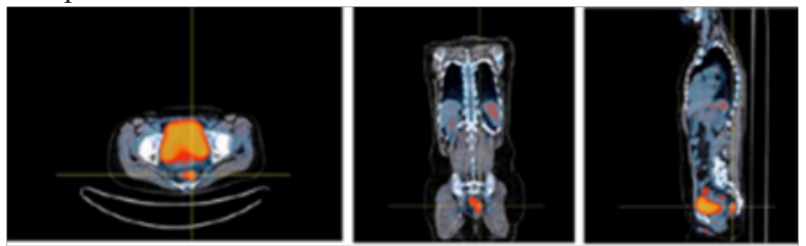

Figure 6 Axial, coronal and sagittal views, after application of FCN, revealing high uptake of I8F-FDG in bladder, due to physiologic retention, and metabolic activity in rectum due to inflammation.

\section{Clinical case of evaluation for brachytherapy (post- RTX)}

Images PET/CT obtained after pelvic external radiotherapy, and after FCN application revealed, in standard views (axial, coronal and 
sagittal), residual uptake area of ${ }^{18} \mathrm{~F}-\mathrm{FDG}$, related with involution of primary cervical tumor, corresponding to good therapeutic response. It is visible an area of high uptake of FDG coincident with the rectum, compatible with radiation side effects in an adjacent organ, with different and lower tolerance dose rates. This phenomenon can be appreciated more accurately in $3 \mathrm{D}$ visualization mode. In those images (Figure 7) it is obvious the high uptake of ${ }^{18} \mathrm{~F}-\mathrm{FDG}$ in small bowel, compatible with inflammatory effects due to ionizing radiation (radiation enteritis). Comment: Brachytherapy is recommended as component of the definitive treatment of locally advanced cervical carcinoma.18 Selection of patients is a critical issue, with clinicians supporting their decisions on clinical examinations and imaging techniques. The potential of PET/CT in evaluation of lymph nodes inside and outside of pelvis is recognized but PET/CT has also been described as an useful tool in 3D- based adaptative brachytherapy, an interesting concept of brachytherapy, ${ }^{19}$ debated during the last decade, that allows improving dose tumor coverage, without significantly increase dose to organ constraints, namely bladder and rectum, in an optimized and personalized treatment. Furthermore the evaluation of treatment response, with metabolic imaging, immediately after radio chemotherapy, has been demonstrated as capable of predicting outcome, making relevant PET/CT evaluation at this time of the course of malignant cervical disease. ${ }^{20}$
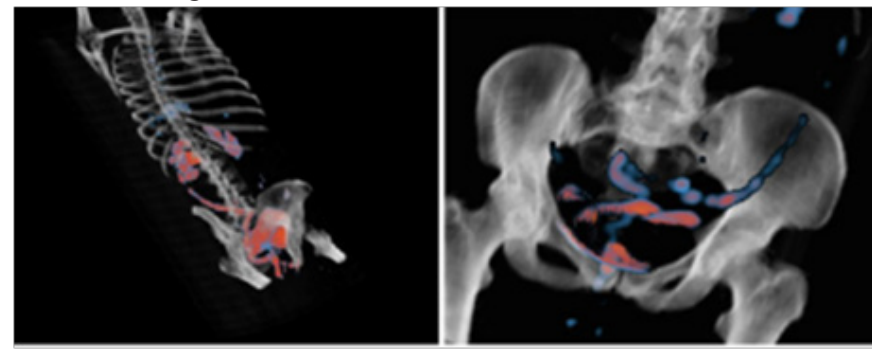

Figure 7 Views in 3D visualization mode after FCN application. Notice high uptake of I8F-FDG in small bowel related to side effects of external pelvic radiation.

\section{Clinical case of follow-up (25 months post therapy)}

Axial, coronal and sagittal views after FCN application revealed multiple focus of high ${ }^{18} \mathrm{~F}-\mathrm{FDG}$ uptake in lymphatic regions, namely, para-aortic and para-tracheal groups (Figure 8). These aspects can be better appreciated in 3D visualization mode, diagnosing exuberant lymphatic dissemination of malignant cervical disease, with apparently local control of cervical tumoral lesion (Figure 9). Comment: Although ${ }^{18} \mathrm{~F}-\mathrm{FDG}$ PET/CT , in current analysis seems to be not cost-effective in the diagnosis of recurrent cervical cancer, as suggested by August et al. ${ }^{21}$ it clearly improves the diagnostic of recurrent cervical cancer, as it provides better anatomic localization of high FDG uptake areas. ${ }^{22}$ Recurrences of cervical cancer are treated with curative intent, mostly when disease is in central pelvic location. Malignant recurrences outside the pelvis are treated with palliative intent but, if recurrence is early diagnosed and treated, it could lead to tumor and symptomatic control, as in our own clinical experience.
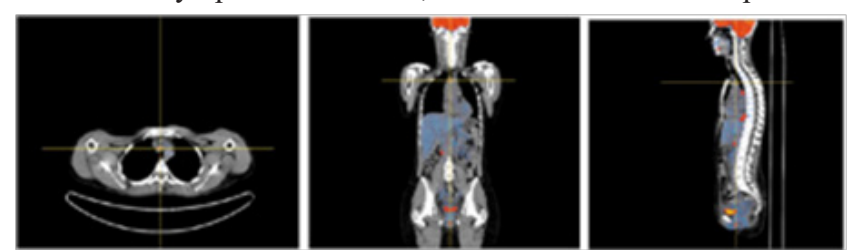

Figure 8 Axial, coronal and sagittal views after FCN application; Axial view reveals high I8F-FDG uptake, related with para -tracheal (mediastinic) lymph node, visible also in sagittal view.

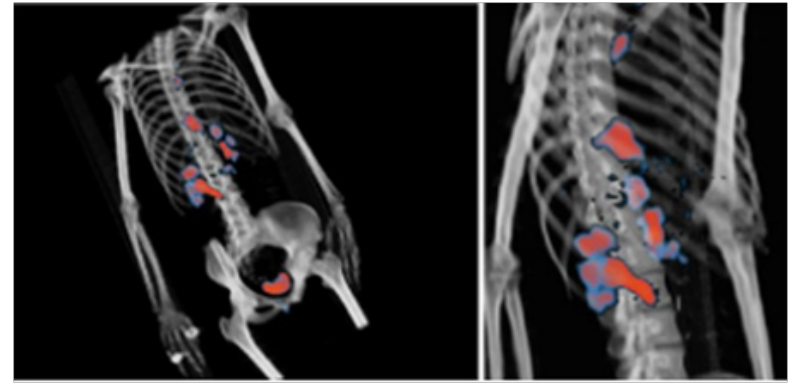

Figure 9 Views obtained from 3D visualization mode, after FCN application, revealed multiple focus of high radiotracer uptake in lymphatic regions.

\section{Conclusion}

Our method of image visualization seemed to be consistent in evaluating areas of ${ }^{18} \mathrm{~F}-\mathrm{FDG}$ uptake, related to metabolism of the primary tumor of the cervix and related to dissemination of the disease through pelvic and para-aortic lymph no. We obtained images of FCN distribution per voxel, corresponding to tumor volume, translating heterogeneous metabolism of ${ }^{18} \mathrm{~F}-\mathrm{FDG}$, matching with the concept of "biological imaging". ${ }^{18}$ We managed to obtain an independent method of visualization that demonstrated improved capabilities to unveil important visual information embedded in the PET/CT datasets. Moreover, our parametric mapping seemed to overpass the subjective quantification of metabolic images, in respect to operator's dependency. It allowed the comparison of exams from different patients and different labs, allowed the comparison of different exams from one patient, in different phases of the disease, avoiding the bladder constraint in exam interpretation, while giving the observer the possibility to interact with images, selecting the best views in each moment. Radiotherapy planning is nowadays a multistep procedure, with sophisticated TPS, using potent algorithms, enabling co -registration of multiple imaging modalities, including functional imaging. The so called biological tumor volume (BTV) is difficult to contour, as it depends on radiation oncologist's choice of methodology, varying from percentage of maximum uptake value, to direct visualization, with the latest being currently used. ${ }^{23}$ On the other hand, the possibility of using advanced techniques of radiotherapy, as IMRT or VMAT, relies on precise delineation of volumes. In cervical cancer, because of invasiveness characteristics of the lesion trough soft tissues, and because of the difficulty to obtain defined margins of tumoral volumes, those techniques are not yet the sate-of -the -art. Our new method of metabolic quantification seems to improve targeting, as it allows an almost perfect visualization of tumor boundaries. This aspect deserves much more investigation and it is clearly a work in progress.

\section{Acknowledgements}

None.

\section{Conflicts of interest}

The authors declare that there is no conflict of interest.

\section{References}

1. Aristei C, Falcinelli L, Palumbo B, et al. PET and PET-CT in radiation treatment planning for lung cancer. Expert Rev Anticancer Ther. 2010;10(4):571-584

2. Delikgoz Soykut E, Ozsahin EM, Yukselen Guney Y, et al. The use of PET/CT in radiotherapy planning: contribution of deformable registration. Front Oncol. 2013;3:33. 
3. Mac Manus MP, Hicks RJ. The role of positron emission tomography/ computed tomography in radiation therapy planning for patients with lung cancer. Semin Nucl Med. 2012;42(5):308-319.

4. Visser EP, Boerman OC, Oyen WJ. SUV: from silly useless value to smart uptake value. J Nucl Med. 2010;51(2):173-175.

5. Keyes JW. SUV: standard uptake or silly useless value? J Nucl Med. 1995;36(10):1836-1839.

6. Boellaard R, Delgado-Bolton R, Oyen WJG, et al. FDG PET/CT: EANM procedure guidelines for tumour imaging: version 2.0. Eur J Nucl Med Mol Imaging. 2015;42(2):328-354.

7. Lindholm H, Staaf J, Jacobsson H, et al. Repeatability of the Maximum Standard Uptake Value (SUVmax) in FDG PET. Mol Imaging Radionucl Ther. 2014;23(1):16-20.

8. Frey EC, Humm JL, Ljungberg M. Accuracy and precision of radioactivity quantification in nuclear medicine images. Semin Nucl Med. 2012;42(3):208-218.

9. Carlier T, Bailly C. State-Of-The-Art and Recent Advances in Quantification for Therapeutic Follow-Up in Oncology Using PET. Front Med. (Lausanne). 2015;2:18.

10. Ferlay J, Soerjomataram I, Dikshit R, et al. Cancer incidence and mortality worldwide: sources, methods and major patterns in GLOBOCAN 2012 Int J Cancer. 2015;136(5):E359-E386.

11. Kidd EA, Siegel BA, Dehdashti F, et al. Clinical Outcomes of Definitive Intensity-Modulated Radiation Therapy With FluorodeoxyglucosePositron Emission Tomography Simulation in Patients With Locally Advanced Cervical Cancer. Int J Radiat Oncol Biol Phys. 2010;77(4):1085-1091.

12. Viswanathen AN. Uterine cervix. In: Halperin, editor. Principles and Practice of Radiatiation Oncology. 6th edn. Walter's Kluver/ Lippincott, Williams and Wilkins, New York. 2013:3501-3713.

13. Cihoric N, Tapia $\mathrm{C}$, Krüger $\mathrm{K}$, et al. IMRT with ${ }^{18} \mathrm{FDG}-\mathrm{PET} \backslash \mathrm{CT}$ based simultaneous integrated boost for treatment of nodal positive cervical cancer. Radiat Oncol. 2014;9:83.
14. Lim K, Small W, Portelance L, et al. Consensus guidelines for delineation of clinical target volume for intensity-modulated pelvic radiotherapy for the definitive treatment of cervix cancer. Int J Radiat Oncol Biol Phys. 2011;79(2):348-355.

15. Haie-Meder C, Mazeron R, Magné N. Clinical evidence on PET-CT for radiation therapy planning in cervix and endometrial cancer. Radiot Oncol. 2010;96(3):315-315.

16. Chao K, Mohan R, Marinetti T. Intensity modulated radiation treatment techniques and clinical application. In: Perez \& Brady, editors. Priciples and Practice of Radiation Oncology. 6th edn. Walter's Kluver/ Lippincott, Williams and Wilkins, New York. 2013:221-246.

17. Lin L, Mutic S, Low D, et al. Adaptative brachytherapy treatment planning for cervical cancer using FDG-PET. Int J Radiat Oncol Biol Phys. 2007;67(1):91-96.

18. Ling CC, Humm J, Larson S, et al. Towards multidimensional radiotherapy (MD-CRT): Biological imaging and biological co formality. Int J Radiat Oncol Biol Phys. 2000;47(3):551-560.

19. Viswanathan A, Thomadsen B. American Brachytherapy Society consensus guidelines for locally advanced carcinoma of the cervix. Part I: General principles. Brachytherapy. 2012;11(1):33-46.

20. Siva S, Deb S, Youn R, et al. ${ }^{18} \mathrm{~F}$-FDG PET/CT following chemoradiation of uterine cervix cancer provides powerful prognostic stratification independent of HPV status: a prospective cohort of 105 women with mature survival data. Eur J Nucl Med Mol Imaging. 2015;42(12):18251832 .

21. Auguste P, Barton P, Meads C, et al. Evaluating PET-CT in routine surveillance and follow-up after treatment for cervical cancer: a costeffectiveness analysis. Brit J Obst Gyn. 2014;121(4):464-476.

22. Ryu SY, Kim MH, Choi SC, et al. Detection of early recurrence with ${ }^{18} \mathrm{~F}$-FDG PET in patients with cervical cancer. $\mathrm{J} \mathrm{Nucl} \mathrm{Med}$. 2003;44(3):347-352.

23. Nestle U, Kremp S, Schaefer-Schuler A, et al. Comparison of different methods for delineation of ${ }^{18} \mathrm{~F}-\mathrm{FDG}$ PET-positive tissue for target volume definition in radiotherapy of patients with non-small cell lung cancer. J Nucl Med. 2005;46(8):1342-1348. 